\title{
A Modified Rational-Function Method for Transmission and Reflection Calculation at Discontinuities
}

\author{
Yih-Peng Chiou \\ Graduate Institute of Electro-Optical Engineering and Department of Electrical Engineering \\ National Taiwan University, Taipei 106-17, Taiwan \\ Email: ypchiou@ntu.edu.tw
}

\begin{abstract}
A novel method based on the Padé approximants is proposed to solve efficiently discontinuity problems. The Padé approximants are derived from the squared charactersitic matrices to avoid the singularities in commonly used approximants. Numerical results show the proposed method is very accurate and efficient, especially for cases with negligiblee evanescent waves in the investigated structures.
\end{abstract}

\section{INTRODUCTION}

Efforts have been made in the calculation of reflection and transmission at discontinuity interfaces which are frequently encountered in laser facets, waveguide ends, gratings, high/anti-reflection coatings, and connections between different waveguides. Such structures can be conceptually depicted a in Fig. 1, an interface $\mathbf{T}$ lying between two media. An incident field launched on the interface from left excites reflected and transmitted fields. Such problem can be solved by modal expansions on both sides and matching the boundary conditions at the interface [1], which works well when the analytical modal information are easily obtained. However, when arbitrary structures are involved, time- and memoryconsuming eigenvalue solvers are normally required in mode finding.

To avoid the resource-consuming eigenvalue solving procedures, Padé-approximant-reflection method with complex reference index (PARM-C) [2],[3] was proposed to efficiently solve such problems. The Padé approximation is a commonly adopted technique in wide-angle beam propagation method (WA-BPM) [4]. In both the WA-BPM and PARM-C the square roots of the derived characteristic matrix $A$ are replaced with their corresponding Padé approximants around a reference index $\bar{n}$. Instead of adopting real reference index in the WABPM, the PARM-C adopts complex reference index to avoid the singularities and to include the evanescent waves [3]. We call the PARM using real reference index as PARM-R to distinguish them. Since the PARM-C can be directly combined with the popular beam propagation method (BPM), multiinterface structures like gratings can also be easily solved [5].

In this paper, we derive the Padé approximants, called PARM-Q, from another operator, the fourth root of the square of the characteristic matrix, $\sqrt[4]{A^{2}}$, instead of $\sqrt{A}$. PARM-Q keeps excellent accuracy of PARM-R for propagating waves. In PARM-Q, the evanescent waves are modeled as if they

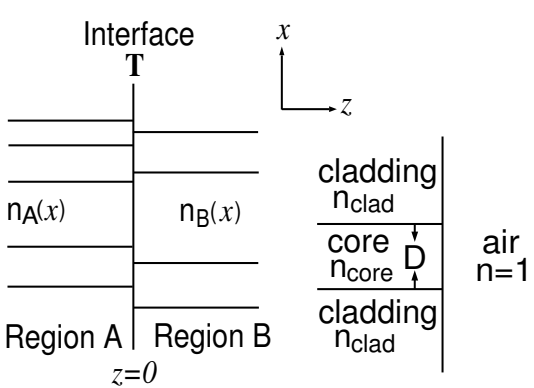

(a)

(b)

Fig. 1. Layered media $A$ and $B$ with refractive index profiles $n_{A}(x)$ and $n_{B}(x)$, respectively, separated by the interface $T$ in the transverse direction.

are the propagating waves. Consequently, the singularities in PARM-R are technically avoided.

\section{THEORY AND FORMULATION}

The two-dimensional Helmholtz equation of a timeharmonic transverse-electric (TE) wave in lossless media $A$ and $B$ can be written as

$$
\frac{\partial^{2} E}{\partial x^{2}}+\frac{\partial^{2} E_{y}}{\partial z^{2}}+k_{0}^{2} n^{2}(x) E_{y}=0,
$$

Considering $z$ propagating waves and discretizing the $x$ direction, we have

$$
\frac{\partial^{2} E}{\partial z^{2}}+A E=0
$$

where $A$ is the characteristic matrix and $E$ represents the column vector composed of electrical fields after discretization. The reflected and transmitted fields at an interface can be written as

$$
\begin{aligned}
& E_{A}^{-}=\frac{\left(\sqrt{A_{A}}-\sqrt{A_{B}}\right)}{\left(\sqrt{A_{A}}+\sqrt{A_{B}}\right)} E_{A}^{+} \\
& E_{B}^{+}=\frac{\left(2 \sqrt{A_{A}}\right)}{\left(\sqrt{A_{A}}+\sqrt{A_{B}}\right)} E_{A}^{+}
\end{aligned}
$$

where $E_{A}^{+}, E_{B}^{+}$, and $E_{A}^{-}$are the incident, transmitted, and reflected electric fields, respectively [2]. The reflected and transmitted transverse-magnetic (TM) waves can be obtained similarly [3]. Usually, the square root of the characteristic matrix $A$ is solved by $\sqrt{A}=X \sqrt{D} X^{-1}$ in such eigenmode 
expasion method as the method of lines (MoL) [6] where $\Lambda$ is a diagonal matrix with its diagonal elements being the eigenvalues of $A$ and the columns of $X$ being the corresponding eigenvectors. These eigenvalues and eigenvectors of the characteristic matrix are in fact the propagation constants and the modes of the structures. The eigenmode expansion method is very accurate but takes tremendous computation time and memory resources, in which the computation memory and time for $N$ nodes are normally $O\left(N^{2}\right)$ and $O\left(N^{3}\right)$, respectively.

To avoid solving the eigenvalue problem, Padé approximants were adopted to approximate the square roots of the characteristic matrices, $\sqrt{A_{A}}$ and $\sqrt{A_{B}}$. The computation time and memory are reduced to $O(N)$. The Padé approximants have singularities, which may result in instability in computation. For example, $(1,1)$ approximant of $\sqrt{A} \approx$ $S_{11}=k_{0} \bar{n}\left(3 A+k_{0}^{2} \bar{n}^{2}\right) /\left(A+3 k_{0}^{2} \bar{n}^{2}\right)$, has a singularity at $-3 k_{0}^{2} \bar{n}^{2}$ if $\bar{n}$ is real. The singularity is avoided if the $\bar{n}$ is complex. In BPM calculation, only propagating waves are involved, therefore, there are no singularities. However, in the calculation of discontinuity problems, the evanescent waves are included, and the singularities will be excited even with tiny amplitudes. Therefore, complex reference indices were introduced to avoid the singularities [2].

In addition to PARM-C, there is another way to avoid the singularity by using real $\bar{n}$, we can also derive the Padé approximants from $\sqrt[4]{A^{2}}$ [7], which is called PARM-Q. The imaginary wave numbers of evanescent waves are squared to become real numbers, and the singularities disappear even with real $\bar{n}$. Mathematically, the evanescent waves are modeled as propagating waves due to $A^{2}$ operation. Therefore, if the evanescent waves are negligible, PARM-Q can be adopted. The propagating modes are more accurately modeled in PARM-Q than that in PARM-C due to the use of $\bar{n}$, which will be shown in the following section.

\section{NUMERICAL RESULTS}

Fig. 2 shows the relative error between the exact propagation constant and the approximated from PARM-Q with Padé approximant of different orders. The singularities due to evanescent waves, corresponding to imaginary $\beta$ or negative $\beta^{2}$, disappear as expected. Propagation constant $\beta$ close to $k_{o} \bar{n}$ are approximated very well like that in PARM-R; that is, the propagating waves are very accurately modeled.

In Fig. 1(b), a waveguide is terminated in air. Evanescent waves may be excited in the air region [2]. With PARM-C, the reflected and transmitted fields can be efficiently solved. If the air is replaced with a dielectric, say, $n_{\operatorname{med} B}=2$, the evanescent waves are less important, and PARM-Q can be applied. Fig. 3 shows results with incident field being the fundamental mode in the left waveguide. The MoL method is used as the reference since it generally give accurate results. The results from PARM-Q22 agree well the MoL method. Our simulation also shows the results are very accurate when the air replaced materials with higher refractive index, which will be demonstrated in conference along with other details.

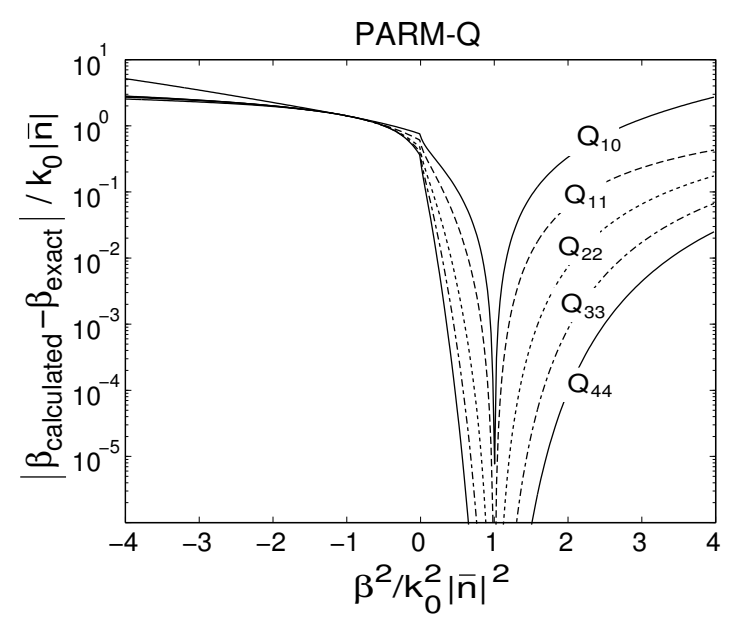

Fig. 2. Relative error in the propagation constant with respect to the square of the propagation constant for Padé approximants of $\sqrt[4]{A^{2}}$ :

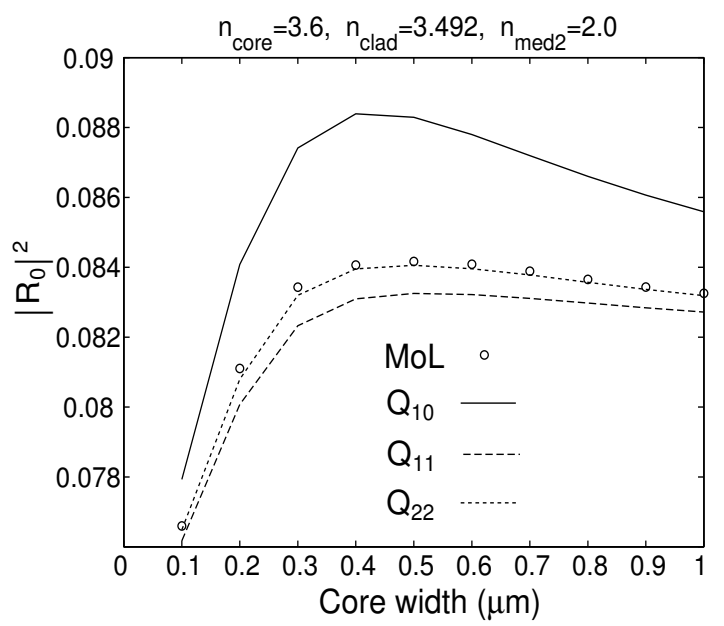

Fig. 3. Reflectivity of the fundamental mode as a function of the core width.

\section{CONCLUSION}

We have proposed a modified Padé-approximant-based method to efficiently solve discontinuity problems. Such method can not only avoids the singularities in Padé approximation but also achieves very high accuracy in modeling the propagating waves. We also demonstrate its applicability with waveguide structures.

\section{ACKNOWLEDGMENT}

This work was supported by the National Science Council, Taiwan, under Grant NSC93-2215-E-002-039.

\section{REFERENCES}

[1] C. Vassallo, J. Opt. Soc. Am. A, 15, 717 (1998).

[2] Y.-P. Chiou and H.-C. Chang, Photon. Tech. Lett, 9, 964 (1997).

[3] Y.-P. Chiou, Novel Techniques in the Finite-Difference Analysis of Optical Waveguides, PhD dissertation, Taipei (1998).

[4] G. R. Hadley, Opt. Lett., 17, 1426 (1992).

[5] H. Rao et al.,Photon. Tech. Lett, 11, 830 (1999).

[6] R. Pregla and E. Ahler, Electron. Lett., 29, 1845 (1993).

[7] G. A. Baker and P. Graves-Morris, Padé Approximants, 2nd ed., New York: Cambridge Univ. Press (1996). 